\title{
ACOUSTIC PENETRATION AND IMPACT DETECTOR FOR MICROMETEOROID AND SPACE DEBRIS APPLICATION
}

\author{
H. Kuczera, H. Iglseder, U. Weishaupt, E. Igenbergs \\ Technische Universität München, \\ Institut für Luft-und Raumfahrt, \\ München, F.R.G.
}

\section{ABSTRACT}

In the past the study of interplanetary dust particles (and probably also small-sized space debris) has mainly been restricted to the measurement of a few parameters like flux, momentum and velocity. Information about the chemical and isotopic composition could be obtained from some Brownlee particles which were collected in the upper atmosphere. An important step has been done with a new experiment on LDEF (Long Duration Exposure Facility) which is still in orbit (launch: April 6, 1984).

LDEF provides the first opportunity to collect micrometeoroid material in space which, afterwards, will be subject to isotopic analysis in the laboratory

The LDEF capture cell experiment for chemical and isotopic measurements of micrometeoroids by secondary ion mass spectrometry will for the first time enable a differentiation between real cosmic dust and man-made space debris, preferably small-sized particles of solid rocket engine exhaust. Information concerning debris flux and size distribution is now of rapidly increasing interest due to the high production rates in current space activities.

The simulation experiments which were performed for the development of the capture cell design and the calibration of the involved analysis instrumentation revealed the necessity to have more information about the impact parameters, such as the impact location on a capture cell, velocity and projectile mass. As a result of these measurement efforts an active detector has been developed with which all required impact parameters can be obtained, and, in addition, the flight path direction of a projectile can be calculated.

The measurement principle is illustrated in Fig. 1. A thin penetration foil is mounted at a distance $d$ above the target plate. This foil is stretched and glued to a support frame. Four piezo microphones, one at each corner, will detect the acoustic bending waves which have been originated by a projectile penetrating the foil. This front foil should be very thin in order not to destroy the particles during penetration, even at high velocities. A metallic coating of one or both sides of the foil, due to electrical shielding reasons or some chemical analysis premises, is still acceptable.

The target plate will be a solid metal plate (with a thickness up to a few millimeters) with another four microphones attached to the rear side. The edges of the target plate are embedded in silicone rubber in order to get sufficient acoustic insulation from the mounting structure. The damping behaviour of the plate can be significantly improved if the rear side is covered with a thick layer of silicone rubber, too. Thus, the signal decay times will decrease and the frequency of detectable events will increase. 
An impacting particle originates a circular bending wave propagating towards the positions of the microphones at the front foil and - if penetration occurs - at the target plate, too. Thus, the arrival times of the acoustic waves according to the different propagation lengths are given.

The evaluation of the impact locations at the foil and at the target plate can easily be performed if always the differences of wave propagation times for two opposite microphones are taken into consideration. These curves for constant time differences are hyperbolae. This overdesigned measurement setup (three microphones would be sufficient for a definite destination) allows a simple and redundant evaluation procedure for the impact location and the event time.

This procedure is schematically shown in Fig. 2. The propagation speeds of the acoustic waves in the front foil and in the target plate can be measured experimentally. With these values and the dimensions of the detector only hyperbola geometry has to be used in order to get the impact location and the event time $\left(\mathrm{P}_{1}-\mathrm{P}_{3}\right.$ and $\mathrm{P}_{2}-\mathrm{P}_{4}$ are the focal distances of the hyperbolae). This detector principale has extensively been tested in the Munich Plasma Accelerator Facility. For the LDEF impact simulation tests the microphones have been attached to the rear-side of the original Capture Cells in order to detect the impact location. In despite of the multi-layer construction (aluminium plate, silicone glue, germanium plate) a sufficient signal-to-noise ratio could be obtained. This has mainly been achieved by an optimization of the microphone set-up.

As an example, the time-of-flight record of three impacts at only an aluminium target is shown in Fig. 4. The time differences $\Delta \mathrm{T}_{13}$ and $\Delta \mathrm{T}_{24}$ can be calculated and - by use of a corresponding diagram as depicted in Fig. 2 - the impact locations can easily be evaluated within an accuracy of $<1 \mathrm{~mm}$. This accuracy is defined by the sample rate of the recording instrument. A similar sequence of signals will be measured for the front foil if a two-stage detector is used.

The Two-Stage Acoustic Penetration and Impact Detector is a simple device for measuring the impact event time, the projectile velocity, the flight path direction and the momentum. The results of the laboratory tests have shown that this detector can be used in a wide range of projectile size and velocity. According to measurement purposes the size of the detection area, the distance between the front foil and the target plate and the number of microphones as well as the evaluation procedure can easily be adjusted. The target plate area can also be replaced by another foil detector, if two penetration stages are preferred.

This active detector is well suitable for a variety of applications in meteoroid and space debris exploration. It can also be supplied with capture cell properties for chemical analysis of inside-deposits.

Therefore, this measurement principale has been taken into consideration as a possible flight experiment for instance for a later LDEF flight or future space station activities.

Reference:

H.Kuczera, H.Iglseder, U.Weishaupt, E. Igenbergs "Two Stage Acoustic Pentration and Impact Detector for Meteoroid and Space Debris Application". Adv.Space Res. Vo1.5, No. 2, pp.91-94, 1985 


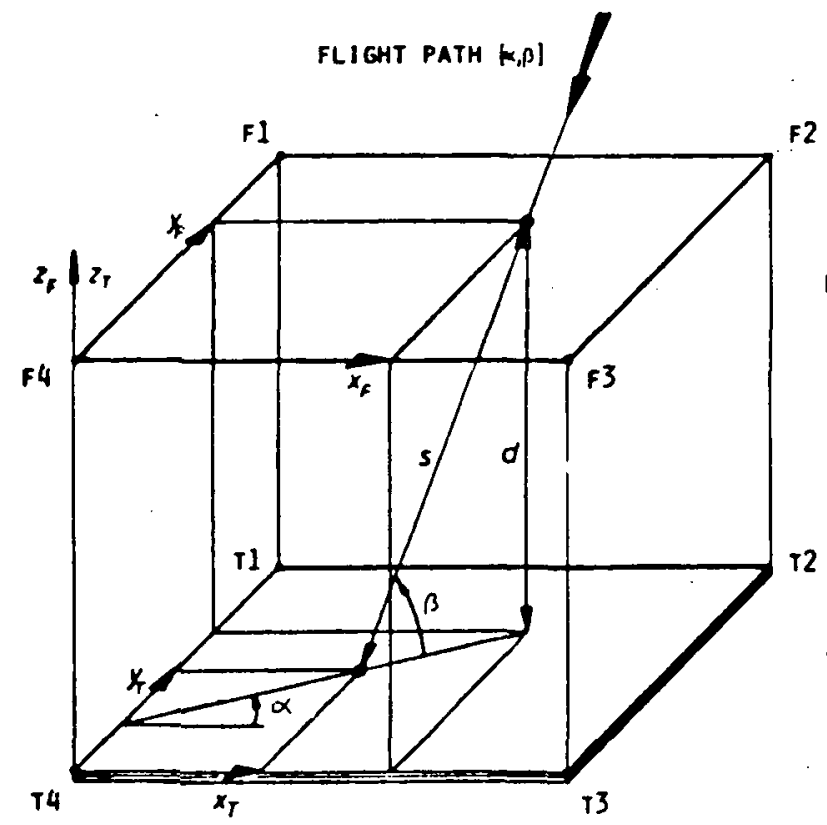

PENETRATION FOIL

TARGET PLATE
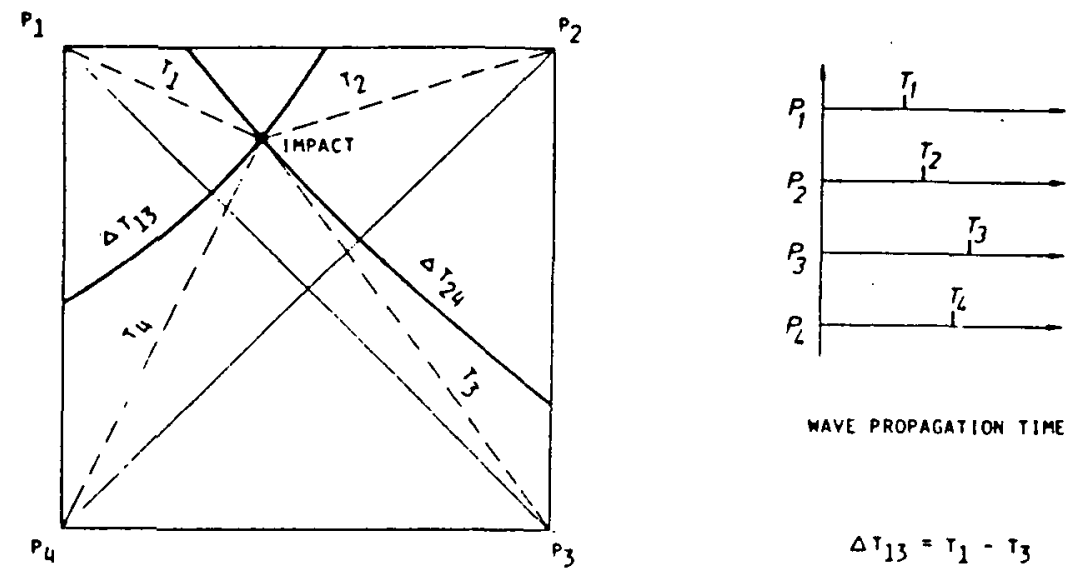

Fig. 1

Detector Principle

$P_{1 . .4}=$ PIEzO MICROPHONES

$\Delta T_{13}=r_{1}-T_{3}$

Fia. 2

Evaluation of the

$\Delta T_{24}=T_{2}-T_{4}$

1

1

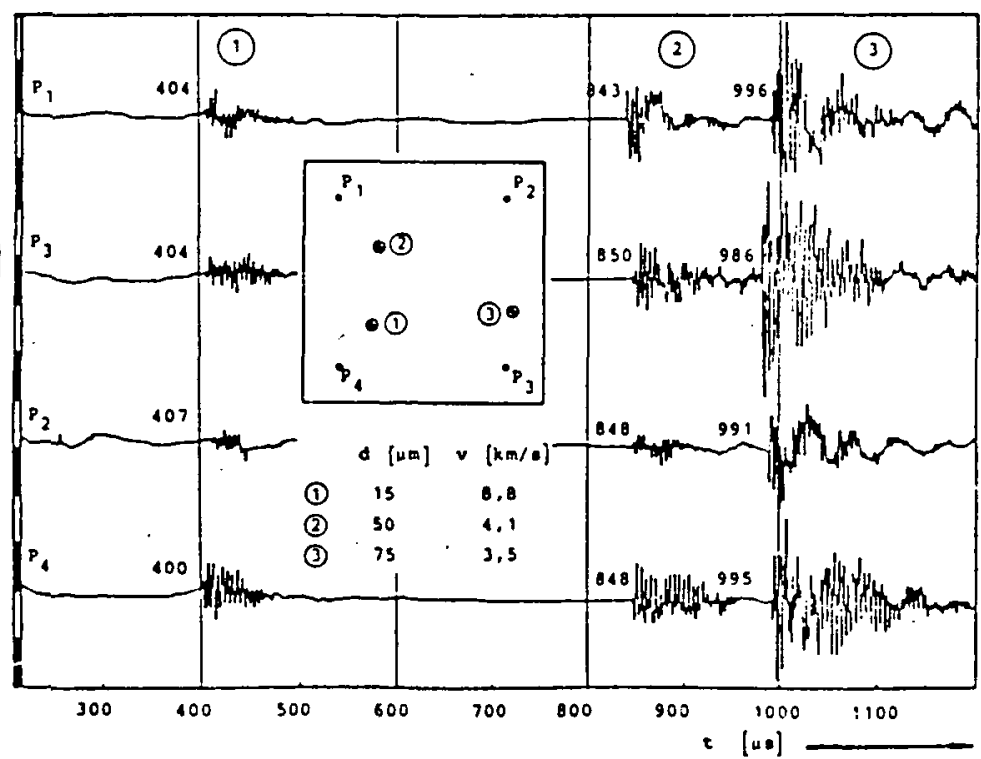

Impact Location

\section{Fig. 3}

Time-of-Flight

Record for a

Target Plate with

three Impacts $(t=0$ :

Trigger of the

Plasma Gun) 